for two or three days but this was probably due to the large doses of arsenic and passed off directly the drug was omitted. This is in striking contrast with most cases recorded, in which the classical symptoms of stricture of the large intestine have led to the diagnosis of cancer and operative measures. At the necropsy there was no evidence of old obstruction.

3. Everything points to this being a primary infection of the mucosa of the cæcum. There were no traces of present or past pulmonary infection and no scarring. It is true that there were pleural adhesions but these were not apical but, rather, generally diffused; possibly these occurred when the patient had inflammation of the lungs at 11 years of age.

Judging by primary tuberculous lesions elsewhere the amount of hyperplasia points to the lesion having been of no recent date. This is also supported by the calcareous and caseous condition of the mesenteric glands. The swelling of the glands of the neck only began to be noticeable six months before the patient's first admission to hospital-that is, two and a half years before his death. The limitation of ulceration to the creum and lower part of the ascending colon with the great hyperplasia causing strictures is in marked contrast with the usually active and extending ulcers due to sputum infection. The lungs showed no scar of any past tuberculous lesion and there was no evidence of bronchial glandular affection, so that general infection could not have taken place by that route. Probably this was a primary infection of the intestinal mucosa, limited to the cæcum and ascending colon, causing secondary extensive infection of the mesenteric glands and later extending to other parts of the lymphatic system-namely, the cervical and axillary glands.

The actual healing of so extensive an area in the intestine, together with the quiescent state of the mesenteric glands, indicates either a low vitality of the particular breed of organism or a high resistance of the individual host's tissues. That the patient should have been able to continue his work with large softened glands in the neck which had certainly been there for nearly three years confirms this.

Numerous cases of so-called hyperplastic tuberculosis of the cæcum have been recorded from time to time. König ${ }^{1}$ records five cases in which operation was performed for symptoms of obstruction. In three of these there was evident pulmonary tuberculosis, whilst of the other two in one there were no signs of disease elsewhere during life and in the other, who died, several ulcers were found in the cæcum, the mesenteric glands were caseous, the bronchial glands were calcified, and the spleen was amyloid. Crowder ${ }^{2}$ gives an account of a case, with a valuable résumé of the literature up to that date. He collected 77 cases, 36 males and 41 females. He refers to the difficulty of diagnosis from malignant disease of the cæcum. The same writer ${ }^{3}$ records a similar case commencing in the vermiform appendix.

Sheffield.

\section{REMARKS ON THE AFTER-TREATMENT OF CASES OF SUPRAPUBIC CYSTO- TOMY, WITH A DESCRIPTION OF A NEW DRESSING FOR THE PURPOSE.}

BX G. H. COLT, M.B., B.C. CANTAB. HOUSE SURGEON TO ST. BARTHOLOMEW'S HOSPITAL.

THe after-treatment of cases of suprapubic cystotomy is not at present entirely satisfactory. The object of this paper is to bring forward what is believed to be a new dressing for a patient with a sinus leading from the suprapubic region to the interior of the urinary bladder. As a house surgeon to St. Bartholomew's Hospital I have had several opportunities of observing the course of, and the effects of treatment on, such fistulæ until the recovery of the patient and it will be convenient to recount briefly some of these observations before proceeding to the main object in view. This will also serve to point out the requirements

1 König: Zeitschrift fuir Chirurgie, 1892, Band xxxiv., p. 65, Die Stricturirende Taberculose des Darmes und ihre Behandlung.

3 T. R. Crowder: American Journal of the Medical Sciences, 1900, p. 668, the Pathology of Chronic Hyperplastic Tuberculosis of the

3 Ibid., 1902, p. 236. expected of such a dressing and to show how these are fulfilled by the device to be described.

The common conditions for which the urinary bladder is opened above the pubes are (1) prostatic hypertrophy with obstruction, (2) vesical calculus, and (3) vesical papilloma and malignant disease. Whatever the condition for which the operation is performed the subsequent method of procedure is nearly always as follows. The edges of the wound in the bladder are sutured above, below, and laterally to the anterior abdominal wall with catgut. These sutures do not pass through the skin. The skin incision is closed with the exception of a hole through which a drainage tube is inserted to the bladder. It is best if this bole fits closely round the tube. The latter is either single, with no lateral holes in it save in the part inside the bladder, or it is a two-way tube. For the present purpose the details of the tube need not be described. It is usually fixed with a stitch which passes through the substance of the rubber only and does not enter the lumen of the tube. The wound is dressed, sometimes a catheter is tied in, and the patient is returned to bed. So far all is good. The wound is dressed as occasion requires and the tube and bladder are washed out. As soon as the main collection of blood, blood clot and débris ceases to appear in the tube and on the dressings-very often before this stage-the tube is geared by a glass union to a second long rubber tube which leads to a receptacle beneath the bed, the end of the tube being beneath the surface of a measured quantity of lotion into which the urine drains away by syphonage, aided, perhaps, by a Sprengel pump or Cathcart apparatus. It is usually unnecessary to wash out the bladder at this stage unless cystitis is present, with perhaps thick urine loaded with mucus which blocks the tube. As a rule about the fourth day it is found that the syphon does not act and the dressings have to be changed every two hours. On inspecting the wound the edges are seen to be everted and granulating round the tube which can be withdrawn smoothly and without sticking. Syphonage can be continued for a day or two longer provided one packs carefully and closely round the tube with a dressing which will prevent the entry of air, such as lint spread with boracic ointment, but the slightest leakage of air into the bladder, and so into the tube, annuls further syphonage action. The urine then only flows over into the tube by its own pressure and more commonly leaks around it. In some cases leakage occurs from the first and cannot be stopped. Attempts to continue the syphonage so often fail that the patient is generally more inconvenienced than benefited by them. In some cases the exact opposite occurs, the tube refusing to work at first but commencing to do so about the fourth day. The tube is usually removed about this time, for there is then little danger of extravasation of urine into the cellular tissue of the pelvis, and it is said that the longer it is retained the longer will the wound take to heal. But it seems that the speedy closure of the wound depends much more on the relative position of the original wounds in the abdominal wall and bladder respectively, other things being equal, than it does on the length of time the tube is retained, provided this be not excessive. For instance, if the incisions in the abdominal wall and bladder are opposite each other the wound takes longer to heal. If, however, after incising the abdominal wall the incision into the bladder is made, not directly opposite the first incision, but an inch or more, as retraction and the nature of the operation allow, towards the orifice of the urethra the final fistula closes the more rapidly. It is, in fact, when first established a straight hole, held straight by a tube along a radius drawn from the centre of the bladder; but directly the tube is removed there is probably a slight sliding of one end over the other and the shear helps to close the fistula. Those who perform suprapubic cystotomy often could easily test the truth of this, which is at present based on such a small number of cases that it is little more than a conjecture. However this may be, the tube is soon removed for good and so are the stitches from the skin. They usually become loose after the six th day. The patient is now in the following condition. He has a nearly healed skin wound and, in some part of it, a complete urinary fistula. Sometimes he has a catheter permanently in the bladder and urethra, but if not one is generally passed from time to time to insure that the natural opening is patent, especially after prostatectomy. If he has no catheter tied in all his urine passes by the fistula. His dressings and bandages are continually soaked and require to be changed every three hours at least, the draw 
sheet being changed at the same time. The urine also help to contribute to the solution of the problem in a flows round beneath the dressings to his back and down the scrotum; his skin is always wet from about the level of the third lumbar vertebra to a point four inches down the thigh. He has to be continually awakened for the dressings to be changed, though usually the wetness itself wakes him. In many cases it is necessary to keep his skin well covered with ointment lest the soaking help to produce sores, and his position in bed must be frequently altered to relieve any long-continued pressure on any bony prominence. Even if he has no cystitis his bed tends to smell offensively, but with the care that is bestowed on him by the sister and nurses in a good hospital or home this is reduced to a minimum. His dressings at hospital price cost about $£ 15 s$. per week; only an approximate estimate is possible. If the patient has a catheter tied in his soaking condition is mitigated somewhat but it is not completely relieved; the external opening of the fistula is closely strapped so that it may heal by third intention but very often the strapping becomes loosened by urine and oscasionally leakage occurs outside the catheter. The proceeding, in fact, seldom hastens recovery as far as can be judged. Although the patient has to put up with this discomfort the results of operations on the urinary bladder performed with proper attention to detail are, as a rule, so eminently satisfactory, afford such immense relief, and tend to such great prolongation of life that it is well worth his while to endure it for a time. There is hardly any class of patient that is more grateful to the surgeon. The fact that all will eventually be satisfactory need not lessen the desire to try to ameliorate his uncomfortable state meanwhile. The condition continues for a very variable time, during which the patient, if at all sensitive, is literally in a state of misery and those who have care of him are often overworked. To illustrate the duration: one fistula healed in 11 days. In this patient the valvular method of opening the bladder described above had been used. Several healed about the seventeenth day, more between the seventeenth and the thirty-fifth day, and some took six weeks or more. One patient had his fistula for four months with offensive cystitis. He was an alien and could not be made to understand and remember what was required of him or to assist his own cure in any way, such as by allowing a catheter to remain fixed in position. This patient died. In the more fortunate cases, and they are the rule, the patient at or about the time the fistula closes passes at first half an ounce of urine per urethram; then, a few hours later, two ounces with a strain and the fistula opens again perhaps. 12 or 24 hours later he passes six ounces and from that time onwards all his urine comes through the urethra. If he gets up under four or five days from the first closing of the fistula the opening often becomes patent and has to close again, advised to be content with his bed or the sofa for a week longer, whereby he will not really lose any time in getting well.

It is obvious from the foregoing remarks that there is very great need of some kind of dressing for application to the patient during the period which elapses between the removal of the skin sutures and tube and the closing of the urinary fistula. This time is at least one week; it is generally about three weeks and often longer still. The requirements are: (1) a dressing which shall be applied on the surface of the skin and which shall not enter the fistula at all-which shall not, therefore, tend to prevent it from closing or cause harm in any way; (2) a dressing which shall collect faithfully all the urine flowing by the fistula and allow it to drain away to a receptacle without wetting the patient's skin; (3) a dressing which shall in no way inconvenience the patient either by its bulk or by causing irritation of the skin or by its attachment to parts remote from the wound as might very reasonably be necessary; (4) a dressing which shall only need occasional inspection and attention, just so much as may also be necessary for proper attention to the edges of the fistula, when, for instance, the granulations become exuberant; (5) a dressing which shall be transparent and so allow the wound to be easily seen; and last of all (6) a dressing which shall be easy of application, simple, unlikely to go wrong, and which shall also be cheap, aseptic, and easily cleaned and sterilised. Such a dressing has been arrived at and is described later; but it will be convenient to state the steps which have led to its being devised, for these may be of some assistance to others working at the subject, by showing the train of thought pursued, and may 
around the orifice of the fistula was shaved, it was swabbed with ether to remove all grease and then kept perfectly dry by soaking the urine up with wool as it appeared on the surface. The dressing having been boiled was thoroughnly dried and coated on its under surface with rubber solution. The patient's skin was also covered with an even thin layer rubbed on well. When the two surfaces had become nearly dry and sufficiently "tacky" the central swab was removed and the dressing was quickly applied. The process was like that employed in mending a puncture in the inner tube of a bicycle tyre, only the patient's skin was not sandpapered. The tube was connected with a receptable beneath the bed. This dressing remained in place for four days and was perfectly efficient in all respects to outward appearances. The patient remained dry externally, the dressing moulded itself to his skin as it wrinkled, he sat up. slept his seven hours at a stretch, and altogether was greatly pleased. The glass union showed that the syphon was full. On the fifth day the dressing leaked and had to be removed, though it would have been removed at this stage in any case to see how the wound was progressing. Then a curious condition was found. For three days betore small sloughs had appeared in the glass pail under the bed, and it was not clear why they should have been there for none had previously been present on the dressings. In the centre of the orifice of the fistula, and having apparently entered the tube, was a conical slough. The base of the slough was a quarter of an inch in diameter and immediately surrounded by a thin blue shelving edge of epithelium. The apex was three-eighths of an inch high. The slough had blocked the tube and performed an experiment which we verified later by clipping the drainage-tube itself. For when the slough had blocked the tube, even though, as we knew in this case, there was no urethral obstruction, the patient had strained and the thin edge of the urinary wedge had inserted itself between the skin and the dressing and had quickly separated a passage.

It is obvious from this trial that an application of sheet rubber stuck on with rubber solution is not in itself alone sufficient for use in sealing up a urinary fistula in all cases should one wish to do so. However, if it is slightly stretched before it is put on it is the best application known at present, and it is likely to be successful in many cases, especially when it is possible to combine it with continuous drainage of the bladder through a catheter, and perhaps also with a truss to make pressure on the layer of rubber.

In this case it was also evident that the syphonage action was far too powerful. Although it was a fall of two feet of water only it had been sufficient to suck a cone of tissue into the wound. This cone looked like granulation tissue and part of it was dead, but it might have been partly mucous membrane. The interior of the bladder was one inch away from the skin when measured by a bent probe. Unfortunately the cone was not submitted to a microscopical examination but the point could easily be settled by repeating the trial. In this respect then the dressing was not only not advantageous but was an actual disadvantage, for skin epithelium will not grow quickly over little mountains such as this cone was. Moreover, the fistula was still quite patent at the base of the cone. This was the objection discovered to the direct sucker principle and referred to before. The remedy was obvious-namely to reduce the length of the long arm of the syphon. This was done down to only one inch of fall, yet the conical slough appeared in 16 hours. The syphonage was then entirely abolished by making a hole in the upper part of the tube near the fistula, thereby allowing the urine to flow over by its own pressure alone. When this dressing was removed two days later no slough was present.

After these preliminary experiments the six requirements mentioned above were reconsidered and ultimately the present apparatus was arrived at. Several forms were tried and the description applies to the one which proved the most satisfactory. The question is a collective one and when one part of the apparatus is altered it is at the expense of the whole. Therefore it is proposed to consider the following device as final until time and experience suggest a permanent modification of it in any particular.

The dressing consists of two parts, one being made of clear glass and the other of sheet rubber. The glass portion is a lightly blown hollow cylinder five-eights of an inch in diameter and modified in that the top is partially closed and the bottom turned outwards as a flange. From the cylinder juts out a tube at right angles to its axis. The accompanying drawing and section offer sufficient explanation. The sheet rubber portion is a circular lisc of the best, most flexible and elastic, untearable, black sheet rubber, three inches in diameter and one thirty-second of an inch thick. It has a central circular hole five-sixteenths of an inch in diameter and can therefore be easily sprung over the glass flange and arranged to lie in continuity with the concave portion of it, as shown in the section. This is important for the proper application and stability of the apparatus. The joint so made is absolutely watertight. The two pieces when adjusted together and to a patient lying on his back
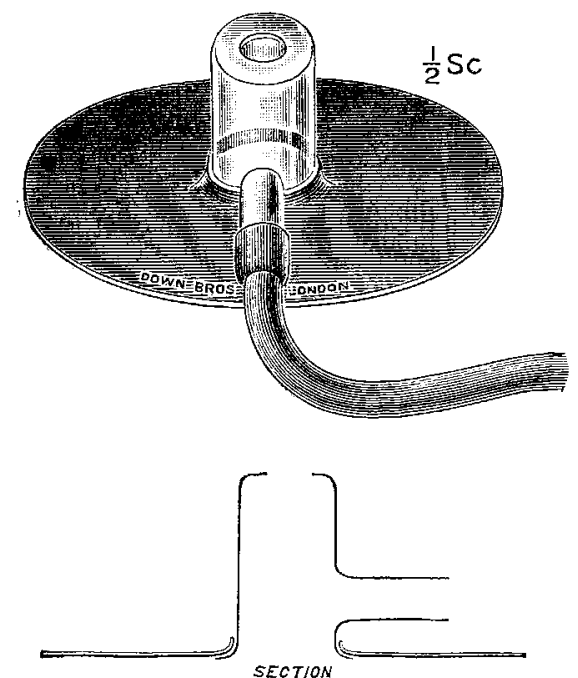

are situated so that the cylinder is vertical and the rubber sheet horizontal. The total weight is three drachms. A larger size is provided for use in the early stage if necessary. The dimensions are a compromise of several, each of which has its own advantages and disadvantages.

The rubber solution employed is made by dissolving pure rubber in naphtha. It is put up in tins and not in tubes. A wide tin is obviously far better for the purpose than a tube. It is highly inflammable and the solvent quickly evaporates, both good reasons for keeping it securely sealed up.

The rationale of the dressing is as follows. Here is a transparent glass vessel open below. It has a hole at the top through which air can enter freely and through which there is access to the opening of the fistula. It has also a tube through which urine can drain off and which can be connected up to a receptacle. It can be attached to the patient's skin by means of the rubber disc and some rubber solution. It has a margin of safety in the extra and seemingly unnecessary height of the cylinder in case the exit tube gets slightly blocked. Urine often rises in this cylinder above the level of the exit tube, even when the latter is on the side on which the patient is lying. The two parts of the dressing are sterilised separately and the application is made as follows. The skin of the patient is shaved over an area of two inches all round the external opening of the fistula. It is then cleared of grease with a swab soaked in ether. The tin of rubber solution is opened and the surgeon sterilises his hands. $\mathrm{He}$ dries them and both parts of the dressing thoroughly with a sterilised towel or with wool, fits the two parts of the dressing together correctly, and adapts the long rubber drainage-tube to the short connecting arm. He causes an assistant, or the patient himself, to keep a small swab of absorbent wool on the orifice of the fistula so that the skin around may be dried and kept perfectly dry until the dressing is applied. This swab should be renewed quickly if it becomes soaked, and it should be small enough to allow the rubber solution to be applied close up to the opening. The surgeon dips his finger in the rubber solution and thoroughly coats the patient's skin with a thin even layer right up to the opening and round it for a distance of two inches, rubbing the solution well in. Care should be taken not to continue the rubbing if the solution becomes "tacky" and begins to peel off. The under surface of the rubber disc is then covered all over with solation but not within one-eighth of an inch of the glass flange or the spring of the rubber is weakened and thereby also the security of the watertight joint. The 
rabber flange bends back of its own accord into three most convenient folds like a three-cornered hat. Then the surgeon waits until the two surfaces are nearly dry and quite "tacky" as tested with the finger. That on the skin takes the longer and it is immaterial how dry the surface of the rubber is. To refuse to wait is to court failure. As soon as the correct moment arrives he causes the central swab to be removed for good and then places the glass flange around the opening of the fistula and quickly, before any urine can escape, presses the rubber disc evenly down all round. An intelligent patient at this stage places his finger on the top of the glass of his own accord. In making the adjustment it should be remembered that the drainage-tube should either point towards the pubes with the rubber tube arranged for the side on which he lies, or it should be turned oneeighth of a circle or so to that side. There should be no attempt at rectification when once the application has been made. The excess of solution is rubbed off the skin round the disc with the finger. It comes off readily enough in little pellets while it is still damp with the solvent. The rubber tube is now bent round in a curve and fixed to the skin by a broad band of leuko. plast midway between the anterior superior iliac spine and the orifice of the fistula, and in such a manner that the glass is kept in correct position partly by the elasticity of the drainage-tube itself; that is to say, there should be no "cocking" of the glass in any direction. The top of the glass is lightly covered with a piece of gauze or wool, or the hole is lightly filled, to prevent sepsis. The drainagetube is threaded through the cradle if one is being used and allowed to hang into the pail. It should be as short as can be contrived. The patient should be instructed to raise up any bed-clothes he may have on the dressing when the nurse has to change them or attend to him, otherwise ihey will catch in it and it will be swept off. He should also be told not to strain to pass urine. When carefully applied the dressing is usually efficient for at least four days. If urine leaks under the rubber the dressing should be removed by peeling it cff from the edge, another rubber substituted, and the whole reapplied. It is generally impossible to readjust it without reapplying it as a whole, though often a strip of plaster applied over the rubber will prevent leakage for one day longer. Ether will dissolve the leukoplast.

The process has taken much space to describe. It takes five minutes only to demonstrate. A few more remarks are necessary. In the first place the patient should not turn far over on the side away from the rubber drainage-tube. If he does so the level of the urine rises above the level of the upper hole in the dressing and the urine flows over. This can be easily prevented by changing the drainage-tube to the other side of the bed, or by having a second tube led off from the cylinder on the other side of the patient, but, with care, the latter is an unnecessary complication and has been discarded. In the second place when he settles himself for sleep he should just see that there is no kink in the tube and that he is not lying on it, as he is nearly sure to be doing if it is fixed too near the ilium. It is far more convenient for all concerned if he lies on an air cushion.

The dressing can also be applied, if necessary, to patients with gall-bladder or other fistulæ. Messrs. Down Bros. Limited, who stock the dressing in a case complete with instructions, send also a suitable modification of it for the gall-bladder cases. It is only necessary, however, to employ this modification when the opening of the fistula is on the side, as distinct from the front, of the abdomen. If the patient with a suprapubic fistula is careful he can wear his dressing while up and about, with the drainage-tube geared to a portable urinal. In this case he wears a dressing similar to that for the gall-bladder fistula. If a catheter is being used as a permanent drain it can be geared by a $T$ union into the one drainage-tube. The bladder can be washed out without disturbing the dressing and its presence greatly facilitates the proceeding. Continuous irrigation in a case of cystitis is also easily arranged.

I am greatly indebted to Mr. D'Arcy Power for having allowed me to use the dressing on some of his cases at St. Bartholomew s Hospital. In conclusion, it may be advisable to state that the above device is only the best of several trials. It fulfils in a simple manner all the requirements of the case. It has been proved to be efficient and I hope it will prove of real practical value

St. Bartholomew's Hospital, E.C.

\section{A CASE OF INTRATHORACIC DERMOID CYST. ${ }^{1}$}

By H. BATTY SHAW, M.D. LoNd., F.R.C.P. LoND,

ASSISTANT PHYSTCIAY TO UNIYERSITY COLLEGE HOSPITAL AND THE HOSPITAL FOR CONSUMPTION AND DISEASES OF THE CHEST, BROMPTON ; AND

G. E. O. WILLIAMS, M.B., B.S. LoNd., LATE HOUSE PHYSICIAN, UXIVERSITY COLLEGE HOSPITAL.

THE patient, a woman, aged 26 years, was admitted as an out-patient to University College Hospital on June 5th of this year. She complained of cough, of the expectoration of "pink" phlegm and hairs, and of wasting and shortness of breath. She had suffered from "weakness of the chest" ever since childhood and at the age of 12 years she was treated for six weeks for left-sided pleurisy and bæmoptysis, and the chest was explored with a needle high up on the left side. Cough had been present for several years; it had been accompanied by a moderate amount of expectoration, on an average less than half a cupful in 24 hours, and for the last three months had been tinged with blood. Hair had been coughed up or "came into the mouth" on hundreds of occasions during the previous eight years. Wasting, shortness of breath, and night sweats had developed only during the previous six months. There were no special features in the family history. The patient was admitted to the hospital under the care of Dr. J. S. Risien Russell, to whom we are indebted for permission to make use of the clinical records.

On admission the patient was found to be rather thin but she did not look ill. The temperature was $99 \cdot 4^{\circ} \mathrm{F}$., the pulse was 90 , and the respiration-rate was 28 to the minute. There was a scar on the nose left after the cauterisation of a wart. There was a suspicion of clubbing of the fingers. The chest was well formed, the right side near the sternum was a little more prominent than the left; slight pulsation was occasionally seen in the second left intercostal space near the sternum. The movements of the chest were good and equal. Vocal fremitus was feeble on both sides of the front of the chest. The percussion note was impaired both above and below the right clavicle, becoming almost absolutely dull over the prominent area and very impaired below as far as the fifth rib, as far out as the anterior axillary line to the right and to the midsternum to the left. The breath sounds were weak above and below the right clavicle and over the very dull area were quite absent; vesicular breathing was heard over the remainder of the right side of the chest. Vocal resonance was well marked above the right clavicle but was much diminished over the dull area. There was slight impairment of the percussion note in the right interscapular region below the level of the spine of the scapula but there was no alteration of breath sounds, vocal fremitus, or vocal resonance in this area. No adventitious sounds were heard over any part of the right chest. The left half of the chest was quite normal. The heart was not dislocated, the apex beat being defined in the fourth space just internal to the left nipple line; there were no murmurs. The liver dulness was normal. On June 27th the patient coughed up two hairs in the presence of a nurse : they were short and coarse and resembled axillary hair; the hairs were unaccompanied by sputum. On July 4th similar hairs were coughed up with some sputum. The sputum was examined on two occasions and was found free from tubercle bacilli ; it consisted mainly of pus and a few fat globules. Extraction with ether showed absence of fat. During the patient's stay in hospital her temperature never exceeded $99^{\circ}$ A radiographic picture, taken by $\mathrm{Mr}$. $\mathrm{E}$. $\mathrm{S}$. Worrall, radiographer to the hospital, showed opacity over the dull area above described, well defined above, below, and to the outer side. but merging into the sternal opacity on the inner side. There were no signs of teeth, bones, \&c. The patient was discharged unrelieved and the symptoms and signs have since continued much the same.

Reports on cases of intrathoracic dermoid cysts are sufficiently rare to justify the publication of an account of this one. The authentic cases number 35 ; some of them are fully reported clinically and pathologically; others are

1 The patient was shown at a meeting of the Clinical Society of London on Oct. $27 \mathrm{th}, 1925$. 